\title{
Diriger un hypogroupe : évolution du management, et des modes de contrôle
}

Managing a hypogroupe: evolution of the modes of management and controls

\section{Caroline Debray}

\section{(2) OpenEdition}

\section{Journals}

Édition électronique

URL : https://journals.openedition.org/travailemploi/5691

DOI : 10.4000/travailemploi.5691

ISSN : 1775-416X

Éditeur

DARES - Ministère du Travail

Édition imprimée

Date de publication : 15 juin 2012

Pagination : $39-55$

ISSN : 0224-4365

\section{Référence électronique}

Caroline Debray, «Diriger un hypogroupe : évolution du management, et des modes de contrôle »,

Travail et Emploi [En ligne], 130 | avril-juin 2012, mis en ligne le 01 décembre 2014, consulté le 29 juillet 2021. URL : http://journals.openedition.org/travailemploi/5691 ; DOI : https://doi.org/10.4000/ travailemploi.5691 


\title{
Diriger un hypogroupe : évolution du management, et des modes de contrôle
}

\author{
Caroline Debray $\left.{ }^{*}\right)$
}

\author{
"Il y a hypogroupe dès l'instant que surgit \\ la volonté de rester en deçà d'une taille critique \\ au-delà de laquelle se poseraient \\ des problèmes de maîtrise. " \\ Michel MARCHESNAY (1991)
}

\begin{abstract}
Entre 2003 et 2008, le nombre de microgroupes recensés par l'Insee a cru de $75 \%$. Pourtant peu de travaux se sont intéressés à ce phénomène significatif. Or, la PME est un champ d'étude majeur qui s'inscrit dans des débats passionnés conduisant «à une lecture plurielle des spécificités du métier de dirigeant" (OBSERVATOIRE DES PME, 2005). Cet article a pour objectif de montrer que l'hypogroupe (MARCHESNAY, 1991), archétype / idéal-type de l'entité statistique microgroupe, est une configuration organisationnelle qui permet de pérenniser la petite entreprise en développement. Par l'étude de la structure de propriété du capital et des logiques d'actions des dirigeants-propriétaires, nous analysons les évolutions des modes de contrôle dans l'hypogroupe, tant au niveau interne qu'externe. Dix cas ont été examinés en 1997. Sur les six hypogroupes encore en activité en 2009 (c'est-à-dire non rachetés ou disparus), quatre ont été retenus afin d'illustrer les modifications du rôle du dirigeant de PME en lien avec l'évolution de la structure de propriété du capital et des domaines de contrôle. À l'instar des dirigeants d'entreprises plus grandes, il recherche une articulation, d'une part entre une professionnalisation du management et une formalisation accrue des modes de contrôle, d'autre part la conservation de caractéristiques spécifiques à la petite taille.
\end{abstract}

En 1987, l'Insee dénombrait 726 microgroupes ${ }^{(1)}$, c'est-à-dire des groupes d'entreprises dont l'effectif total est inférieur à cinq cents salariés ${ }^{(2)}$. De 22174 microgroupes en 2003 (regroupant 54212 entreprises), ce nombre passe à 38755 en 2008 , ce qui représente une augmentation de près de $75 \%$ sur cinq ans et $95,3 \%$ de l'ensemble du nombre total de groupes recensés en France ${ }^{(3)}$. Distinguant des sous-classes d'effectifs ${ }^{(4)}$, Nadine LeVRaTTO (2007) constate que chaque microgroupe est composé en moyenne de quatre entreprises et que plus de $50 \%$ des entreprises constituant ces microgroupes ont

(*) Université Montpellier II - Montpellier Recherche en Management; caroline.debray@iut-nimes.fr

(1) Le microgroupe, comme la micro-entreprise, est une catégorie statistique.

(2) Source Insee : http://www.insee.fr/fr/methodes/default. asp ? page $=$ definitions/microgroupe. htm; page consultée le 5 octobre 2012.

(3) Entre 2007 et 2008, les microgroupes, les petits groupes (de 500 à 1999 salariés) et les moyens groupes (de 2000 à 9999 salariés) ont vu leur nombre augmenter alors que le nombre de grands groupes (plus de 10000 salariés) diminuait de 94 à 91. Source : "Images économiques des entreprises et des

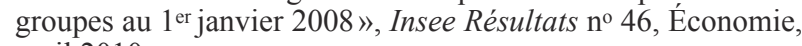
avril 2010.

(4) Soit de $0-1$ à 4 salariés; 5 à $9 ; 10$ à $19 ; 20$ à 49 ; et 50 à 249 . moins de vingt salariés. Or, malgré cette augmentation remarquable du nombre de microgroupes, la spécificité de leur gouvernance et les raisons qui ont motivé leur constitution sont méconnues des pouvoirs publics. En effet, les programmes et les actions qu'ils destinent aux petites et moyennes entreprises (PME) ne font pas de distinction entre les différentes catégories de taille (COMMISSARIAT Général du Plan, 2004; Pacitto, Julien, 2007) et de format d'organisation. Quant à l'Insee, il affirme «qu'une grande partie de ces entreprises contrôlées se comporte très probablement comme des PME de taille équivalente» (KADJAR, 2007, p. 3). Or, divers auteurs s'inscrivent en faux contre cette représentation des microgroupes. Pour Anne Skalitz (2002, p. 2), " agréger ou comparer des entreprises de même taille mais de statut différent - filiale ou indépendante - a peu de sens : leur autonomie de décision, leur stratégie de développement voire de production sont distinctes $»$.

Le succès de ces formes organisationnelles et capitalistiques ne réside-t-il pas précisément dans une souplesse qui leur permet de s'adapter et de survivre, y compris aux cessions et transmissions? Afin de répondre à cette question, nous allons préciser et clarifier la notion de microgroupe. Grâce à des 
caractéristiques juridiques et fiscales empruntées aux grandes entreprises ${ }^{(5)}$, elle apparaît comme une configuration susceptible de pérenniser l'organisation. La structuration sous forme de groupe intervient en général dans des conditions qui rendent une mutation nécessaire, soit du fait de processus de réorganisation (lors d'une transmission familiale par exemple), soit du fait de pressions externes sur l'entreprise (par un donneur d'ordre) ou de la mise en œuvre d'une stratégie de croissance (Mallard, 2004). Dans un contexte de progrès technologique et de globalisation progressive des marchés, la petite taille s'avère être un désavantage majeur, notamment en matière d'innovation, facteur de compétitivité déterminant et coûteux. Qu'il s'agisse de la recherche d'une souplesse de gestion en maintenant une unité de direction (SKALITZ, 2002) ou de l'intensification de la sous-traitance par l'externalisation croissante de l'activité des grandes entreprises générant des cascades de PME sous-traitantes (LOISEAU, 2001), plusieurs facteurs expliqueraient l'essor des microgroupes. Cet essor se caractériserait également par un double mouvement de concentration (au sein de la holding et des filiales) et de déplacement du pouvoir managérial (Aubert, Beaujolin-Bellet, 2004). Comme le soulignent André LetowsKi et Philippe Trouvé (2004, p. 86), «si les modèles d'entreprises supposent une certaine stabilité dans le temps rendant possible leur identification et leur comparaison, ils n'en recèlent pas moins, parfois simultanément, des processus d'hybridation plus aptes à illustrer leur dynamique».

Peu de recherches se sont, à notre connaissance, intéressées à l'analyse de cette trajectoire de développement, aux motivations du dirigeant-propriétaire et aux évolutions en termes de gouvernance, de management et de contrôle qui en résultent. Bengt JoHANNISSON (1990) et Michael SCOTT et Peter Rosa (1996) ont proposé des travaux pionniers en la matière. Or la croissance démographique du nombre d'hypogroupes ces dernières années, dans un contexte de durcissement des conditions de sous-traitance (gestion des stocks, des risques) parfois difficiles pour les entreprises, et alors même que le taux de disparition des petites entreprises (PE) et très petites entreprises (TPE) est très élevé, atteste sans conteste de l'attrait de cette forme organisationnelle, notamment dans le cadre des politiques publiques pour l'emploi (6).

Dans la mesure où, dans l'hypogroupe, la séparation entre la propriété du capital et le contrôle est évitée de manière quasi systématique, il est permis, en première approche, de situer l'hypogroupe au sein de l'unité statistique large qu'est le microgroupe.

(5) Nous citerons entre autres : l'autonomie juridique des filiales, le régime de l'intégration fiscale, le régime des sociétés mère et filles, etc.

(6) Citons par exemple l'exonération des charges pour l'emploi du premier salarié dans une société à responsabilité limitée (SARL), mesure qui a fortement encouragé la création de nouvelles filiales pour les dirigeants interrogés.
Dans une première partie, à partir d'une revue de la littérature, nous définirons l'hypogroupe, idéaltype du microgroupe comme stratégie alternative de croissance de la petite entreprise. Nous retiendrons un effectif par filiale inférieur à quarante-neuf salariés, conformément à la définition européenne de la petite entreprise. Ce choix nous semble pertinent au regard des effets de seuils juridiques et fiscaux qui interviennent à partir de cinquante salariés mais également au regard des constats de Levratto. Nous ferons ensuite un état de l'art sur les travaux traitant des caractéristiques juridiques, fiscales et stratégiques du groupe de sociétés et sur l'intérêt que peut trouver le dirigeant d'une petite entreprise à adopter ce mode de développement. Dans une seconde partie, nous exposerons la méthode qualitative que nous avons retenue et qui est «recommandée lorsqu'on aborde des champs nouveaux, complexes, où les développements théoriques sont faibles et où la prise en compte du contexte est déterminante pour l'élaboration du processus de compréhension » (EVRARD et al., 1993). Nous justifierons également de notre choix d'une typologie sur continuum comme grille de lecture de l'évolution des différentes modalités du contrôle dans l'hypogroupe. Nous présenterons dans la troisième partie une étude de cas comparative menée en 1997 et 2009 sur quatre hypogroupes. Cette étude étaie la thèse de l'article : la nature de la propriété du capital permet de caractériser de quelle manière le(s) dirigeant( $\mathrm{s})$ dirige (nt) et contrôle (nt) l'hypogroupe. Elle repose sur le caractère familial de la propriété (plusieurs membres d'une famille détiennent plus de $51 \%$ des droits de vote) ou personnel (un ou plusieurs associés se partagent le capital).

\section{Du microgroupe à l'hypogroupe}

En opposition avec les théories managériales de l'hyperfirme, nous nous sommes appuyée sur la notion d'hypofirme et la théorie afférente développées par Marchesnay (1982, 1991, 2004, 2008). L'hypofirme se caractérise par des structures peu intégrées (ayant un très faible nombre de niveaux hiérarchiques), une recherche de flexibilité, une aspiration à l'indépendance et à l'intégration socio-économique, au détriment du couple traditionnel «profit-croissance». Définissant l'hypogroupe comme l'archétype de la notion statistique de microgroupe, nous montrons comment le choix d'une structure de groupe permet le développement de la petite entreprise en dehors d'un effet-taille direct, alors qu'il peut également résulter d'une volonté de rationalisation de l'organisation (LOISEAU, 2001), par la filialisation d'activités auparavant intégrées dans la structure.

\section{L'hypogroupe, groupe de petites entreprises}

L'hypogroupe est un groupe, au sens juridique et financier du terme (Lefebvre, 2006), de petites, 
voire de toutes petites entreprises (moins de cinquante salariés), unies par des relations financières qui donnent à l'une d'entre elles, la société mère, le pouvoir de décision sur les autres, les filiales (voir schéma 1). Les pourcentages de détention du capital ci-dessous sont donnés à titre illustratif. La séparation entre la propriété et le contrôle est limitée, voire évitée puisque l'hypogroupe se caractérise notamment par le fait que le dirigeant est propriétaire d'une part importante du capital, ce qui lui confère un rôle décisionnel central. Bien que résultant pourtant d'une pratique entrepreneuriale installée, l'hypogroupe, stratégie alternative de croissance en petite entreprise, est une notion encore théoriquement émergente.

Schéma 1 : Un exemple de configuration d'hypogroupe

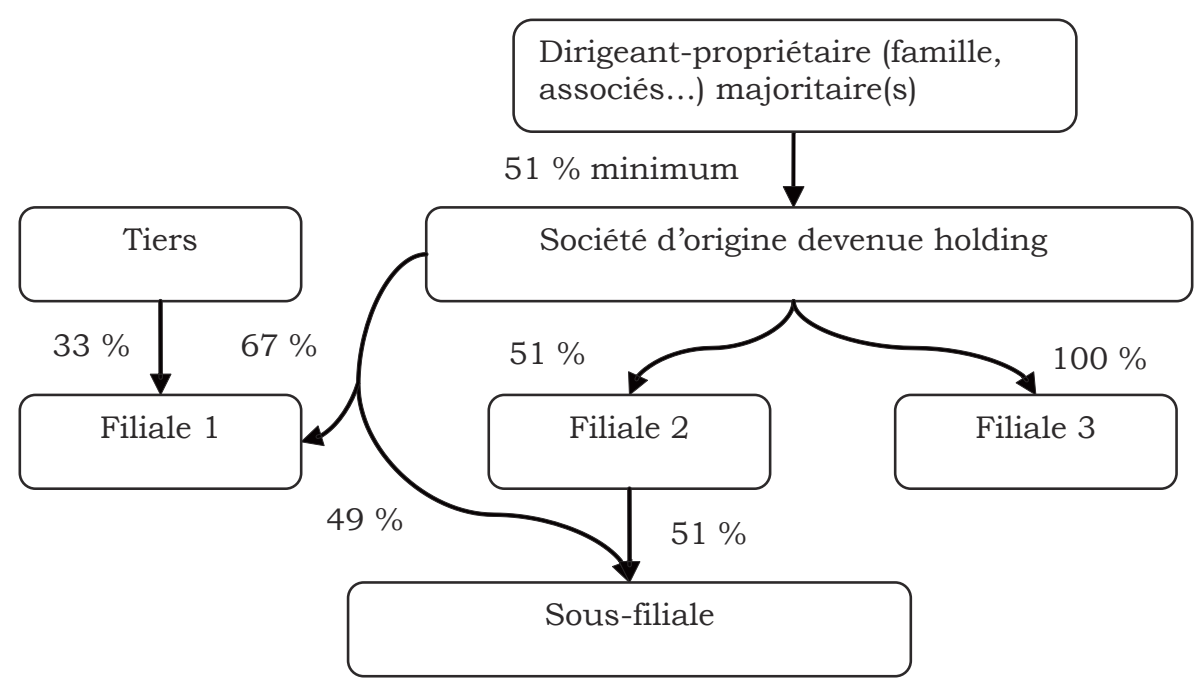

Source : d'après DeBRAY (1997a).

\section{Pour une appréhension nouvelle de la croissance en petite entreprise}

L'analyse et l'hétérogénéité des différents travaux traitant des stratégies de croissance en PME montrent la difficulté d'appréhension globale du phénomène (GibB, Scott, 1983 ; Mc Millan, Lowe, 1986; Perry, 1987; Gasse, CArrier, 1992). Les stratégies de croissance des PME décrites dans la littérature posent de nombreux problèmes, tant sur le caractère réducteur d'une "croissance inéluctable vers la grande dimension » (CHURCHILL, LEWIS, 1983; HANKs et al., 1993; GoDENER, 2002) que sur la non prise en compte de détentions simultanées de petites entreprises par un même dirigeantpropriétaire (DoncKels et al., 1987; KolvereiG, Bullvag, 1992; Birley, Westhead, 1994; Wright et al., 1995). Les travaux sur la détention multiple d'entreprises (dites «propriétés») par un même entrepreneur (dit «habituel» ou « récidiviste») sont relativement récents et peu nombreux (STOREY, 1994; Debray, 1997a, 2000). Le paradoxe entre la croissance exponentielle de cette forme organisationnelle et la quasi-absence de recherches sur cette modalité de croissance des petites voire des très petites entreprises (TPE) peut s'expliquer par le fait que les banques de données fournissent essentiellement des informations centrées sur l'unité statistique «entreprise» (effectif, secteur, statut, etc.). Les difficultés majeures de la recherche dans ce domaine proviennent du manque d'observations et d'évaluations systématiques de la présence de ce type de dirigeants-propriétaires dans l'économie. Pour ScotT et Rosa (1999, p. 22), «la recherche au niveau de l'entreprise ne pourra jamais prendre en compte ce processus entrepreneurial», et se borne à constater simplement que «les petites entreprises ne grandissent pas». En effet, l'entreprise conserve une petite taille et se développe par la constitution d'autres petites entreprises qui lui sont financièrement liées. L'analyse de l'hypogroupe pourra donc être menée de façon pertinente au niveau du détenteur multiple de propriétés et par l'étude de ses motivations pour constituer un groupe de petites entreprises.

\section{Le groupe de sociétés, une stratégie de croissance et de rationnalisation de l'organisation pour la petite entreprise}

De nombreux travaux ont étudié les grands groupes internationaux sous un angle juridique, fiscal ou organisationnel (MonTMORILLON, 1986; BATsCH, 1993), écartant de leur analyse les groupes de PME. Les principales études des phénomènes de contrôle à l'intérieur des grands groupes se sont attachées au lien entre propriété du capital et pouvoir de décision (Charreaux, Pitol-Belin, 1989). Hormis les études de VAN Loye (1991), et de Mathé et Rivet (1993) ${ }^{(7)}$, la population des

(7) Ces études portaient sur des PME d'un effectif moyen de 250 salariés. 
petites entreprises n'a pas fait l'objet d'études cherchant à relier la structure de propriété du capital et les décisions stratégiques. Des travaux tels ceux de BASLY (2006) montrent qu'il y a eu peu de nouvelles recherches sur le lien entre propriété du capital et pouvoir de décision en PME; celles qui ont été menées ont porté le plus souvent sur des populations d'entreprises familiales, particulièrement dans le cadre de recherches sur le financement de la PME (Colot, Croquet, 2005 ; Cirac, 2007; Bughin, Colot, 2008). Pour Davidsson, Achtenhagen et Naldi (2004), les motivations à l'origine de la croissance du groupe trouvent leur origine dans un réseau complexe de modèles de propriété et diffèrent de celles de la grande entreprise. Diverses études ont montré que la structure sous forme de groupe de sociétés pouvait procurer des bénéfices de nature variée. Les avantages juridiques, fiscaux et stratégiques liés au groupe de sociétés (DEBRAY, 2000) permettent à une petite entreprise de poursuivre une stratégie de croissance en dehors d'un effet-taille direct. Elle peut ainsi exercer un meilleur contrôle à la fois de ses approvisionnements (intégration amont) et de sa distribution pour garantir l'écoulement des produits (intégration aval), ou diversifier ses activités. La structure de groupe permet la concentration des efforts concurrentiels de chaque filiale sur des segments ciblés et rend les opérations de croissance relativement réversibles, puisque la revente d'une filiale est plus simple que le démembrement d'une partie de l'activité interne (DEBRAY, 1997a; Mallard, 2004). L'autonomie juridique des sociétés a pour but de réduire les risques financiers, notamment en cas d'échec, tout en facilitant la diversification des activités et en fournissant une structure de responsabilisation de la gestion (émulation-concurrence entre les filiales) souvent plus efficace que pour des activités organisées en départements dans une même société. La transmission au sein d'une même famille est facilitée (BARTHÉlÉmy et al., 1991; DeBray, 1997b) par la création d'une holding familiale par exemple. La surface (i. e. le nombre de filiales) du groupe renforce également le pouvoir de négociation avec un certain nombre de partenaires (banques, fournisseurs, distributeurs). Enfin, la constitution d'un hypogroupe peut se faire par la filialisation d'une activité précédemment intégrée dans la structure, ou par croissance externe, en rachetant des sociétés.

Intégrant et combinant des éléments spécifiques de la petite taille avec celle du groupe, nous avons, lors d'un premier travail portant sur dix hypogroupes (DEBRAY 1997a), élaboré une grille d'analyse du processus d'hypogroupement. Neuf hypogroupes sur les dix étudiés à l'origine ont été créés en transformant la société d'origine en holding et en filialisant une activité (existante ou nouvelle).
Selon que l'hypogroupe est détenu de manière personnelle (par une personne ou plusieurs associés, souvent deux) ou familiale, deux logiques émergent. La logique entrepreneuriale permet à l'hypogroupe de conserver une certaine autonomie de décision et de valoriser les actifs et les compétences dans un développement maîtrisé de l'organisation; l'objectif est alors la revente à plus ou moins long terme, à moins que des événements personnels n'affectent la trajectoire de développement ${ }^{(8)}$. La logique patrimoniale, quant à elle, se caractérise par la recherche prioritaire d'une indépendance financière et une accumulation d'actifs (essentiellement dans un objectif de transmission).

Ces deux logiques ne sont pas contradictoires : elles expriment, par la structure de propriété du capital et un effectif très souvent contenu sous le seuil des cinquante salariés, la volonté du dirigeant de maintenir son entreprise sous contrôle, tant sur le plan interne qu'externe.

En raison des spécificités reconnues de la petite entreprise (indépendance/autonomie, proximité, flexibilité, adaptabilité, effectif, seuils légaux), les choix effectués en termes de contrôle de l'organisation lorsque celle-ci se développe peuvent être très différents. Le dirigeant peut choisir d'internaliser de nouvelles activités au sein de l'entreprise d'origine, ce qui élargit ainsi son espace de commande et diminue le risque de perte de pouvoir. Il peut sinon externaliser une partie de son activité pour préserver les atouts de la petite taille; le corollaire peut être alors une perte de maîtrise des transactions comme dans le cas des TPE «qui ne souhaitent pas s'engager dans des processus de différenciation structurelle susceptibles de remettre en cause une certaine cohérence du système de gestion et une certaine harmonie sociale » (WIKLUND et al., 2003). Enfin, le dirigeant peut choisir de filialiser une ou plusieurs activités, ce qui lui permet de conserver les avantages liés à la petite taille tout en bénéficiant de ceux issus de la croissance de fait de son entreprise (économies d'échelle, de champ ${ }^{(9)}$, d'apprentissage [WALTER, BARNEY, 1991]). Il crée ainsi une structure à la fois isolée de la première mais se situant toutefois dans les frontières de l'organisation qu'il dirige. La croissance de l'organisation n'est alors visible que si l'observateur tient compte du fait que le dirigeant possède plusieurs entreprises via une société-mère alors que l'effectif de chaque filiale n'augmente pas. On note donc une stabilité des effectifs de chaque entreprise alors même que leur

(8) Voir plus bas le cas PROMOTEUR.

(9) Également appelées économies de variétés, il s'agit des économies liées à l'élargissement de la gamme par exemple (qui permet d'amortir les frais fixes plus rapidement en fabriquant des produits complémentaires sur un même outil de production). 
nombre peut croître. En filialisant des activités au lieu de les agréger aux activités existantes (internalisation), le dirigeant cherche à minimiser non seulement les risques liés à une perte de contrôle mais également ceux liés à une mise en dépendance, risques que le choix de l'externalisation (sous-traitance par exemple) fait encourir à l'entreprise (Debray, Leyronas, 1998).

La structure de propriété du capital apparaît ainsi comme un élément structurant de la croissance en petite entreprise (DEBRAY, 2000). Nous nous sommes donc fondée sur la nature des capitaux (personnels ou familiaux) et la structure de la propriété au sein de l'hypogroupe afin de caractériser les relations qui existent entre ces variables et les types de contrôle exercés par le dirigeant d'hypogroupe à deux périodes distinctes.

À mi-chemin entre le courant de la spécificité qui considère que la PME, concept à part entière, n'est pas une grande entreprise miniature et n'a pas, de ce fait, vocation à utiliser les mêmes outils de gestion, et le courant de la diversité qui consiste, en s'appuyant sur des typologies, à ordonner l'hétérogénéité du monde des PME (Torrès, 2004 ; OBSERVATOIRE DEs PMe, 2005, p. 38), nous nous référerons au courant de la synthèse (Julien, MARChESNAY, 1994, p. 14) qui essaie de dégager des typologies, en tenant compte des critiques adressées aux deux précédents courants d'analyse. Le point commun de ces trois courants étant la place centrale du dirigeant au sein du système de gestion, il nous semble dès lors pertinent de resituer le dirigeant de petite entreprise au cœur du processus de croissance qui le contraint, au fil de l'évolution de son organisation sous forme de groupe, à modifier ou adapter ses pratiques managériales, de gouvernance et de contrôle. Nous avons noté des types de comportements à des moments clés du développement qui permettent d'appréhender et de comprendre les trajectoires suivies.

\section{Méthodologie de l'étude}

Toute PME se situe à un point intermédiaire sur un continuum intégrant différentes caractéristiques constitutives de l'hypofirme (Julien, MarchesNAY, 1994, p. 15) et observées dans de nombreuses études faites par les auteurs. Les critères de taille, le type d'activité et de produits (traditionnels ou de pointe), le type de marché (de local et protégé à international et ouvert) et la technologie utilisée (degré d'innovation, procédé et organisation) caractérisent l'entreprise.

Le système de gestion est analysé à travers le degré de centralisation des décisions, l'organisation et le mode de contrôle de l'entreprise (indépendance, appartenance à un réseau ou à un groupe, centralisation, etc.). Enfin, la stratégie est définie par son niveau de formalisation et sa nature réactive ou proactive, de survie ou de croissance. La typologie sur continuum de Julien et MARChESNAY permet de situer et d'observer les évolutions d'une entreprise donnée sur un ensemble de facteurs de contingence définissant la PME de manière quantitative (effectif) et qualitative. Nous l'avons adaptée à l'hypogroupe et avons développé les variables liées au contrôle de l'organisation afin de caractériser de manière approfondie l'évolution dans le temps des liens entre la propriété du capital et les modes de contrôle, interne et externe, dans l'hypogroupe.

\section{Une démarche essentiellement qualitative}

Nous avons retenu une méthodologie essentiellement qualitative car, d'après BRYMAN (1984), l'étude de cas est «plus pertinente que l'approche quantitative dans l'étude des phénomènes organisationnels dynamiques et humains ». Par une analyse en profondeur des perceptions et des comportements humains (JAusCh et al., 1980 ; Miles, HuberMAN, 1988; YIN, 1994), l'étude d'au minimum quatre cas (voir encadré) permet de faire ressortir les variables qui ont une influence sur la question à l'étude (Wacheux, 1996). Cette démarche s'avère nécessaire dans la mesure où «il n'existe malheureusement pas de théorie unitaire qui offre un guide adéquat, que ce soit pour un modèle de développement, ou pour la production d'hypothèses prenant en compte les caractéristiques des entrepreneurs habituels ${ }^{(10)} \gg$ (STARR, BYGRAVE, 1992).

L'analyse des cas à plusieurs années d'intervalle permet de montrer en quoi la trajectoire personnelle du dirigeant (par exemple, son souhait de pérenniser son entreprise par la transmission à un héritier) peut avoir un effet sur la structure de la propriété du capital de l'hypogroupe, sa logique d'action et son développement. Le nombre d'années écoulées entre 1997 et 2009 induit certaines limites, notamment des biais liés aux perceptions des dirigeants ainsi qu'à la rationalisation a posteriori des manœuvres de croissance ou de recentrage. La préparation de l'étude en 2009 s'est notamment faite en renvoyant au dirigeant, préalablement à l'entretien, l'analyse de 1997 qu'il avait validée ${ }^{(11)}$.

(10) Entrepreneurs habituels et récidivistes sont considérés ici comme synonymes; il s'agit ici de la détention simultanée, par un même dirigeant ou une même famille, de plusieurs entreprises.

(11) Les dirigeants ont relu et validé nos analyses établies d'après les entretiens. Il s'agissait de s'assurer de la cohérence de notre analyse avec le discours du dirigeant. 


\section{Encadré \\ Méthode d'enquête}

Des entretiens approfondis, utilisant une grille d'analyse systémique du processus d'hypogroupement (DEBRAY, 1997a; voir annexe 1) ont été menés en 1997 et 2009 auprès de dix dirigeants-propriétaires d'hypogroupes (1). Cette grille d'analyse s'appuie sur les quatre piliers du système de gestion de la PME et intègre également le cheminement de la croissance et les éléments structurants de la forme de groupe (DEBRAY, 2000). Les entretiens, d'une durée variant de 1 heure 30 à 3 heures ont été enregistrés et retranscrits dans leur intégralité. Ils ont permis de mettre en évidence les objectifs poursuivis par le dirigeant dans le choix d'une structure de groupe pour faire face au développement de sa petite entreprise, les perspectives envisagées (transmission, revente), le degré d'instrumentation (2) du contrôle interne comme externe. Nous avons ensuite procédé à la codification des données lors de l'analyse des grilles. Les hypogroupes œuvrant dans des secteurs variés (industriels et/ou de services), cela nous a permis de faire ressortir des caractéristiques intrinsèques au secteur d'activité. Chaque grille d'analyse ainsi construite a été soumise aux dirigeants à des fins confirmatoires en 1997 et 2009. Quatre cas sont présentés dans cette étude, choisis pour leur représentativité, au regard des logiques d'action suivies par les hypogroupes et de la nature de la propriété du capital, ainsi que de leur évolution entre les deux études. Les variables étudiées ont ensuite été resituées sur une typologie sur continuum.

Le recueil nécessaire de données structurelles et quantitatives nous a également amenée à administrer un questionnaire. Soixante et onze questions, ouvertes et fermées, administrées en face-à-face, nous ont permis de définir le périmètre des hypogroupes, de formaliser leurs configurations en termes de structure du capital et de propriété sur la base des variables du contrôle figurant en annexe 2 . Ils ont également permis de reconstituer l'évolution factuelle des hypogroupes.

Une analyse documentaire des moyens et instruments de contrôle utilisés dans chaque hypogroupe (sociétémère et filiales) a été menée. La nature parfois peu formalisée des outils de pilotage a pu être explicitée grâce aux entretiens.

(1) Sur les dix cas étudiés lors de la première vague d'étude, un hypogroupe a disparu suite à une mésentente entre héritiers; un autre, spécialisé dans les biotechnologies, a été racheté par un géant de l'industrie agrochimique.

(2) Le degré d'instrumentation représente l'intensité de l'utilisation d'outils formalisés permettant l'analyse puis la prise de décision (Nobre, 2001).

\section{La typologie sur continuum : une grille de lecture des modes de contrôle}

Nous nous sommes interrogée sur la possibilité de caractériser les relations qui existent entre la structure de propriété du capital (personnel ou familial) et les types de contrôle exercés par le dirigeant d'hypogroupe (Debray, 2009). Nous avons ainsi mis en regard certaines caractéristiques spécifiques de la gestion de la petite entreprise, telles que le rôle central de l'entrepreneur (LECOINTRE, 2010), la recherche d'indépendance dans la prise de décision à travers une organisation souvent peu formalisée, et une stratégie souvent implicite (MARCHESNAY, 1997), et un management de proximité (TORRÈs, 2003) avec des indicateurs de contrôle repérés dans la littérature. Présentés en annexe 2, ces derniers ont pour objet la compréhension des modes de fonctionnement interne et de contrôle de la structure.

Définir la notion de contrôle dans l'hypogroupe revient à apprécier le degré de pouvoir du dirigeant sur son organisation; celui-ci se traduit par la détention majoritaire du capital dans la holding, des participations majoritaires de la holding dans les filiales et l'attribution par le dirigeant en personne des postes-clés de direction et de contrôle (holding et filiales). Au niveau interne, les variables retenues sont le mode de direction des filiales (concentré ou délégué), le degré de formalisation des procédures de contrôle, les modes de coordination et le degré de délégation. Au niveau externe, le contrôle est caractérisé par le niveau d'intégration de l'hypogroupe au sein de la filière, par exemple s'il a racheté un fournisseur ou un distributeur. La typologie sur continuum du schéma 2 synthétise les différentes variables du contrôle de l'hypogroupe. Chacune peut être spécifiée à un instant t et visualisée par un curseur mobile sur chaque flèche.

Schéma 2 : Typologie sur continuum de l'hypogroupe

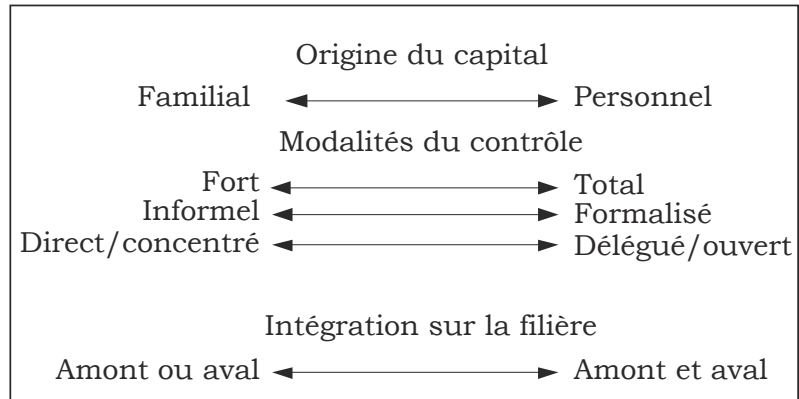

Source : Debray (2010).

\section{Un contrôle interne fondé sur le mode de direction des filiales et les organes de décision}

Le mode de direction des filiales dépend de la catégorie de dirigeants placés à leur tête. On distingue les dirigeants-propriétaires des dirigeants salariés, ainsi que leurs modes de direction respectifs, notés direct et délégué dans les schémas 3 à 6 
(voir plus bas). Le pouvoir de décision du dirigeant est lié à son statut dans la holding, dans les filiales et dans les organes de décision. Le pouvoir est total si l'indépendance financière du dirigeant est complète par rapport à l'actionnariat de la holding. Le dirigeant détient les pleins pouvoirs décisionnels par sa participation dans le capital et les postes de direction occupés (président-directeur général [PDG] dans les sociétés anonymes [SA] ou gérant majoritaire dans les sociétés à responsabilité limitée [SARL] et président du conseil d'administration [CA]). Le pouvoir est fort si des coalitions d'actionnaires minoritaires peuvent gêner le dirigeant dans certaines de ses décisions (notamment en assemblée générale extraordinaire).

\section{Une formalisation plus ou moins avancée des procédures de contrôle}

Deux modes privilégiés de contrôle de l'activité des filiales sont analysés : un contrôle formalisé à l'aide de procédures écrites (tableaux de bords mensuels, comptes-rendus écrits de l'activité commerciale, comptes de résultats mensuels voire hebdomadaires) ainsi qu'un contrôle informel caractérisé par la présence physique du dirigeant dans chacune des filiales ainsi que par des contacts réguliers avec les dirigeants salariés, s'il y en a, ou le personnel d'encadrement. S'il y a des documents-types de contrôle, ils sont peu nombreux et leur fréquence peut être sporadique.

\section{Un contrôle externe traduit par le degré d'intégration dans la filière d'activités}

La répartition de ses activités, sur un ou plusieurs segments de la filière économique, va traduire le niveau d'intégration de l'hypogroupe. Ce niveau d'intégration, qui facilite une répartition et une minimisation des risques liés à la dépendance, explique en partie la logique de constitution de l'hypogroupe. La répartition des activités dans les filiales se fait de deux façons. D'une part, les activités peuvent être réparties par activités dites «productives»; il s'agit d'activités ayant vocation à être mises en concurrence sur le marché externe, mais également à faire l'objet de transactions intrafiliales à des prix de cession internes (voir annexe 1). D'autre part, la répartition peut se faire par fonctions dites « de prestations de services» (comme le support logistique, la comptabilité ou les achats), fonctions qui ont pu auparavant être intégrées à l'organisation.

Nous nous sommes appuyée sur cette typologie pour réaliser les schémas $3,4,5$ et 6 qui synthétisent et décrivent pour chaque cas les évolutions observées entre les deux périodes d'étude. Nous obtenons ainsi des photographies, à deux instants $t$, à la fois des configurations des hypogroupes et des modes de contrôles associés à une géographie des différents pôles de pouvoir (les associés ou autres responsables clés). Grâce à notre grille d'analyse, nous avons resitué les cas traités sur les axes de la typologie sur continuum, en positionnant un «curseur» pour situer chaque variable à l'étude (schémas 3 à 6).

\section{Présentation des cas}

De la structure de propriété du capital (familial ou personnel) de la holding de l'hypogroupe découlent deux logiques d'actions (patrimoniale ou entrepreneuriale) qui dépendent des objectifs poursuivis en 1997. Les quatre hypogroupes que nous décrivons ici illustrent la diversité des trajectoires suivies. Les événements de la vie personnelle des dirigeants vont influencer voire modifier radicalement leurs objectifs poursuivis et leurs logiques d'action, avec pour conséquence une adaptation de la structure du capital afin de leur permettre de conserver la maîtrise de l'organisation hypogroupe. Les résultats ci-après illustrent quatre cas de dynamiques (familial-familial, familialpersonnel, personnel-familial, personnel-personnel).

\section{Les hypogroupes à capitaux familiaux : un objectif prioritaire de transmission}

En 1997, deux hypogroupes, METAL et BTP, sont structurés autour d'un capital familial. L'objectif affiché du dirigeant de METAL est de transmettre la direction à son fils aîné Bruno sans pour autant léser ses deux autres enfants. En 1997, sur les deux frères à la tête de BTP, un seul a un enfant, alors en bas âge. En 2009, la famille s'est agrandie d'un second enfant, mais leurs âges respectifs ne permettent pas d'anticiper leur avenir. Néanmoins, leur père aimerait qu'ils reprennent le flambeau, «s'ils le souhaitent et s'ils en ont la capacité».

\section{METAL : d'un hypogroupe familial à un hypogroupe personnel ou le passage d'une logique de transmission familiale à une logique entrepreneuriale de valorisation}

L'hypogroupe METAL et ses neuf filiales œuvrent dans le secteur de la métallerie (serrurerie) et de la chaudronnerie industrielle (construction de bennes à papiers, cuves à gazole, conseil en équipements pétroliers et stations services, location de matériels et de personnels de chantiers). L'idée de son fondateur en 1997 est de travailler avec ses trois enfants et de leur transmettre à terme son affaire. La holding de METAL est alors détenue majoritairement à parts égales par le père, Joseph, artisan soudeur de son état, et un des fils, Bruno ayant un BEP de comptabilité. Le frère cadet de Bruno gère deux filiales, son oncle est également à la tête d'une filiale et sa sœur occupe un poste dans la filiale qui centralise les achats du groupe. Entre 1997 et 2009, Bruno, seul vraiment intéressé par la gestion du groupe, rachète les parts de son frère et de sa sœur. La constitution d'une holding de famille, dès 1994, permettra à la sœur 
et au frère de Bruno d'hériter du patrimoine immobilier personnel de leur père tandis que Bruno reste «seul maître à bord» de l'entreprise. La première entreprise du groupe est créée en 1978 et produit alors des containers en chaudronnerie industrielle pour des donneurs d'ordre. L'hypogroupe se constitue progressivement par rachats d'entreprises concurrentes, «mais néanmoins amies », en difficulté, dont Joseph s'est engagé à régler les dettes. La plupart restent gérées par leurs anciens dirigeants. Cette stratégie d'intégration de concurrents dans l'hypogroupe permet de renforcer la position concurrentielle sur le marché régional. Bruno, le fils aîné, poursuit cette stratégie de développement et crée de nouvelles filiales dont la vocation est l'accompagnement du développement de l'hypogroupe, «chaque société faisant pour les autres ce qu'elle fait mieux que les autres». La première gère l'ensemble des livraisons du groupe, la deuxième centralise les achats tandis que la troisième commercialise les différents produits du groupe. Développé autour d'un métier de base, la chaudronnerie, l'objectif de constitution de cet hypogroupe est une intégration amont et aval sur la filière et la mise en place de transactions intragroupes palliant les déficiences éventuelles du marché. D'autres rachats (équipements pétroliers notamment) ont fini de structurer le groupe.

Le tableau 1 reprend la structure de propriété, les secteurs d'activités et synthétise les modifications opérées dans le périmètre de l'hypogroupe (évolution du nombre de filiales et de l'effectif global de l'hypogroupe). Entre 1997 et 2009, Bruno «rationnalise ses activités » en revendant certaines filiales (dont un bureau d'études électroniques).

Tableau 1 : Structure du capital, secteur et périmètre de l'hypogroupe METAL

\begin{tabular}{|c|c|c|}
\hline Cas METAL & Situation en 1997 & Situation en 2009 \\
\hline Capital & Familial & Personnel \\
\hline $\begin{array}{c}\text { Secteurs } \\
\text { d'activités }\end{array}$ & $\begin{array}{c}\text { Chaudronnerie } \\
\text { industrielle- } \\
\text { métallerie }\end{array}$ & Inchangé \\
\hline Périmètre/effectif & 9 filiales/85 & 5 filiales/80 \\
\hline
\end{tabular}

Ainsi, en 2009, METAL est devenu un hypogroupe de type personnel (voir schéma 3). Bruno, quarante-quatre ans alors et sans enfant, souhaite pouvoir le revendre avant ses cinquante ans, «afin de réaliser son rêve, vivre au Canada». L'objectif de pérennisation à des fins de transmission a été atteint à la seconde génération. La logique d'action a ensuite évolué puisque la priorité fut donnée à la valorisation des savoirs et savoir-faire afin d'accroître la valeur du groupe à des fins de cession.
Schéma 3 : Déplacement du curseur entre les deux périodes d'étude de METAL

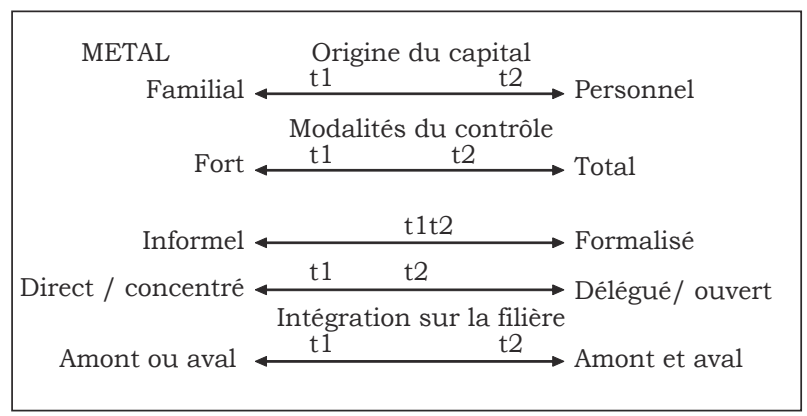

\section{BTP : une logique patrimoniale pérenne dont l'évolution sera soumise à la question des héritiers}

L'hypogroupe BTP, familial également, se développe à partir de 1987 autour de la construction et des travaux publics. Deux frères s'en partagent le capital à égalité (voir tableau 2). André est ingénieur en bâtiment tandis que François est autodidacte. L'hypogroupe se constitue au gré d'opportunités, d'événements ou de transformations de certaines de ses sociétés (comme le changement, la création ou la reprise d'une nouvelle enseigne). En 1997, trois entreprises sœurs constituent l'hypogroupe. Deux filiales construisent et commercialisent des maisons individuelles, premier métier du groupe. Une troisième filiale créée en 1989 est dédiée à l'édification de grands bâtiments (notamment un stade de dimensions très importantes). Lorsque l'opportunité de racheter une agence immobilière se présente en 1998, une quatrième filiale intègre le groupe pour gérer notamment les fichiers clients des deux filiales initiales. La cinquième, provenant d'un rachat, est spécialisée dans l'ingénierie et la recherche de sites sur lesquels construire des bâtiments spéciaux de stockage de produits toxiques. La holding, considérée comme "une coquille creuse», fournit certaines prestations aux filiales, perçoit et réinvestit les dividendes en fonction des besoins de chacune. Selon André, son intérêt principal est de compenser les difficultés conjoncturelles de certaines filiales en permettant «une refacturation des frais de siège». Il applique ainsi un principe de subsidiarité, «source d'efficience». En 2007, la troisième filiale qui gérait les fichiers clients a été revendue à un réseau d'agences immobilières, le groupe BTP souhaitant se recentrer sur son cœur de métier (voir schéma 4).

Tableau 2 : Structure du capital, secteur et périmètre de l'hypogroupe BTP

\begin{tabular}{|c|c|c|}
\hline Cas BTP & Situation en 1997 & Situation en 2009 \\
\hline Capital & Familial (50-50) & Familial inchangé \\
\hline Secteurs/activités & $\begin{array}{c}\text { BTP et promotion/ } \\
\text { construction }\end{array}$ & Inchangé \\
\hline Périmètre/effectif & 6 filiales/167 & 5 filiales/160 \\
\hline
\end{tabular}

Si la bonne entente entre les deux frères a permis jusqu'à présent une gestion sans heurts de l'hypogroupe, cette dernière est-elle pour autant efficace? 
Si les dirigeants reconnaissent le risque de déstabilisation encouru si une mésentente vient à survenir, ils se refusent à tenter de résoudre ce problème qui peut remettre en cause la pérennité de leur hypogroupe d'autant que le temps parle pour eux : cela fait trente ans que cette association perdure sans problèmes majeurs.

Dans l'hypothèse où aucun des enfants ne souhaiterait reprendre l'affaire, l'hypogroupe sera certainement «cédé par branche ou dans sa totalité selon les offres d'acquéreurs».

Schéma 4 : Déplacement du curseur entre les deux périodes d'étude de BTP

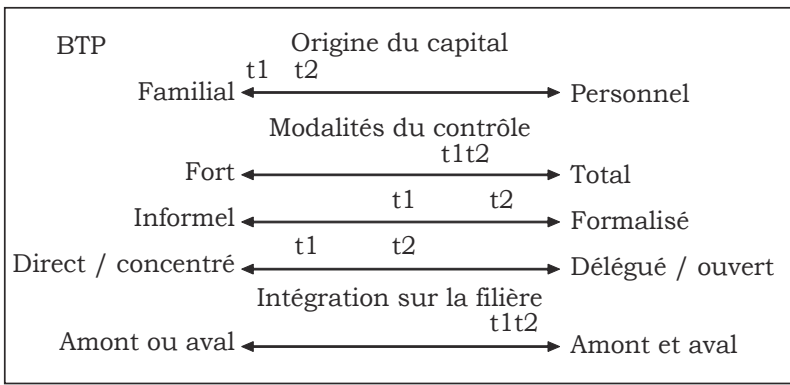

La création d'une holding de famille a pour but, dans les hypogroupes familiaux, de pérenniser l'organisation au fil des générations (BIRLEY, 1986; BARTHÉLÉMY et al., 1991) en permettant éventuellement le désengagement d'un membre de la famille (Debray, 1998, op. cit.) comme ce fut le cas pour METAL. Mais cette solution n'a pas été proposée à BTP par leur expert-comptable. Lors de la première étude, nous avons pu constater que les avocats ou experts-comptables des hypogroupes avaient des compétences hétérogènes sur la question. La mise en place d'un pacte successoral destiné à assurer la stabilité du contrôle de l'hypogroupe relève d'une logique patrimoniale forte et peut être rapprochée de l'objectif de transmission aux héritiers dans deux cas (METAL en 1997 et PROMOTEUR en 2009, voir plus bas).

\section{Les hypogroupes personnels : une logique de valorisation des actifs et des compétences}

Deux hypogroupes personnels poursuivaient des objectifs de valorisation lors de l'étude de 1997. Les histoires de vie vont modifier la logique d'action du premier, PROMOTEUR, tandis que le second poursuit ses efforts de rentablité.

\section{PROMOTEUR : d'un hypogroupe personnel à un hypogroupe familial ou l'influence de la vie per- sonnelle sur la trajectoire professionnelle}

Marc PROMOTEUR est promoteur-constructeur de logements collectifs de standing en Languedoc et Provence-Alpes-Côte d'Azur (PACA). À l'instar de la majorité des sociétés de promotion-construction, son entreprise est structurée sous forme de groupe (personnel). Majoritaire dans le capital de la société de services (Promotion), cette dernière détient une société d'ingénierie financière de projets de construction. Une société civile immobilière (SCI) est créée à chaque programme de construction. Elle joue le rôle de maître d'ouvrage et de gestion du programme de commercialisation. Ces SCI disparaissent lorsque le programme est entièrement commercialisé, en général au bout de quatre à cinq ans. En 2009, les deux filles du dirigeant terminent des études supérieures en commerce et gestion. L'aînée souhaite intégrer l'entreprise. Son père la nomme directrice générale de la holding. La cadette, passionnée de sport, obtient un diplôme en management des organisations de sports et loisirs aux Etats-Unis. Son père rachète et filialise au sein du groupe PROMOTEUR, un $c l u b$ de sport de la région et le transforme en complexe haut de gamme, y intègre des activités liées au bien-être, et place sa fille à sa tête. Par ailleurs, il attribue à chacune d'elles une fraction minoritaire du capital de la holding. En 2009, la structure de propriété de PROMOTEUR est devenue familiale, le père restant PDG (voir tableau 3).

Tableau 3 : Structure du capital, secteur et périmètre de l'hypogroupe PROMOTEUR

\begin{tabular}{|c|c|c|}
\hline $\begin{array}{c}\text { Cas } \\
\text { PROMOTEUR }\end{array}$ & $\begin{array}{c}\text { Situation } \\
\text { en 1997 }\end{array}$ & Situation en 2009 \\
\hline Capital & Personnel & Familial (filles) \\
\hline Secteurs/activités & $\begin{array}{c}\text { Promoteur } \\
\text { constructeur }\end{array}$ & $\begin{array}{c}\text { Activité } \\
\text { additionnelle } \text { : sports } \\
\text { et loisirs }\end{array}$ \\
\hline Périmètre/effectif & 3 filiales/27 & 5 filiales/60 \\
\hline
\end{tabular}

Selon Marc, les répercussions de la crise financière ont provoqué un net ralentissement de la mise en route de nouveaux chantiers. Cela se traduit par un nombre de filiales en 2009 moins important que ce que l'on aurait pu attendre. Le schéma 5 met en lumière l'évolution du capital (qui a eu pour conséquence une délégation de pouvoir plus large) et du mode de contrôle interne entre 1997 et 2009 (des tableaux de bords plus affinés sont désormais utilisés).

Schéma 5 : Déplacement du curseur entre les deux périodes d'étude de PROMOTEUR

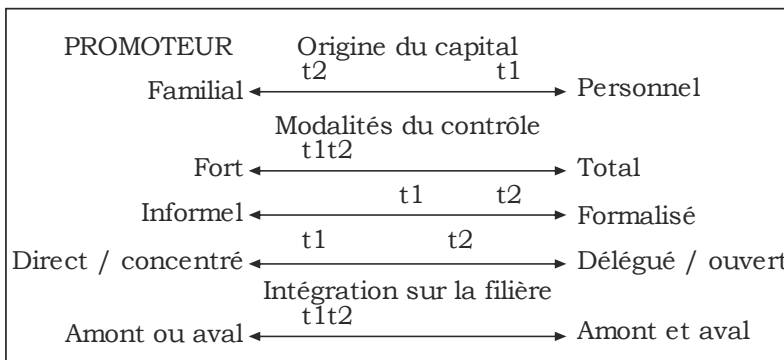

Exception faite de l'addition de l'activité «sport et bien-être», les caractéristiques de ce secteur d'activité expliquent le fait qu'il n'y ait pas eu d'intégration dans la filière. 


\section{ELECTRO : Un hypogroupe personnel mû par} une logique de valorisation forte des compétences et des savoir-faire dans une perspective de cession

Ingénieur physicien de formation, Jean débute sa carrière comme salarié dans deux grands groupes d'informatique et de télécommunications. Suite à l'obtention d'un DESS en gestion, il se retrouve à la direction générale d'une moyenne entreprise. Souhaitant "ne plus avoir de compte à rendre à personne», il crée une première entreprise spécialisée dans l'électrotechnique, puis rachète ou crée des filiales spécialisées dans l'électronique et l'électromécanique (moteurs, gyroscopes, cartes à puces pour la RATP, capteurs de radiations nucléaires, capteurs de répartition du kérosène, armoires hertziennes, etc.). Le développement du groupe s'est, selon ses dires, «toujours effectué sans appel à des capitaux extérieurs $»$ car Jean refuse toute ingérence extérieure dans "son affaire». Son taux d'endettement consolidé, de $21 \%$ (la moyenne du secteur étant à $64 \%$ ), s'explique par le fait qu'il n'ait procédé à aucun versement de dividendes, ce qui serait pourtant avantageux fiscalement. En effet, la détention de chaque filiale à $95 \%$ permet le versement des dividendes en quasi-franchise d'impôt. En dépit de cet argument, Jean souhaite réinvestir tous les bénéfices dans le développement de son affaire afin de limiter le recours à l'endettement (voir tableau 4).

Tableau 4 : Structure du capital, secteur et périmètre de l'hypogroupe ELECTRO

\begin{tabular}{|c|c|c|}
\hline Cas ELECTRO & Situation en 1997 & Situation en 2009 \\
\hline Capital & Personnel & $\begin{array}{c}\text { Personnel et } \\
\text { investisseurs } \\
\text { minoritaires }\end{array}$ \\
\hline $\begin{array}{c}\text { Secteurs } \\
\text { d'activités }\end{array}$ & $\begin{array}{l}\text { Industriel et } \\
\text { commercial : } \\
\text { électronique } \\
\text { et technique- } \\
\text { international }\end{array}$ & Inchangé \\
\hline Périmètre/effectif & 6 filiales/120 & 5 filiales/125 \\
\hline
\end{tabular}

PDG majoritaire à $85 \%$ de la holding, président du CA, il est également PDG des deux filiales sous statut de SA et gérant minoritaire des trois autres. Il n'a pas accordé de délégation aux trois gérants majoritaires de ces SARL provenant de rachats. Jean assure seul le suivi des investissements et le contrôle des filiales à l'aide de tableaux de bords qu'il a luimême élaborés. En 2009, la filiale spécialisée dans le câblage d'immeubles (alors sous gestion de son ancien propriétaire, actionnaire minoritaire), est la seule filiale gérée par une personne extérieure, ingénieur Supéléc; elle est alors revendue à un groupe d'investisseurs de Taïwan car, pour Jean, c'est l'opportunité de "réussir un coup» et de dégager des ressources nouvelles (voir schéma 6).
Schéma 6 : Déplacement du curseur entre les deux périodes d'étude d'ELECTRO

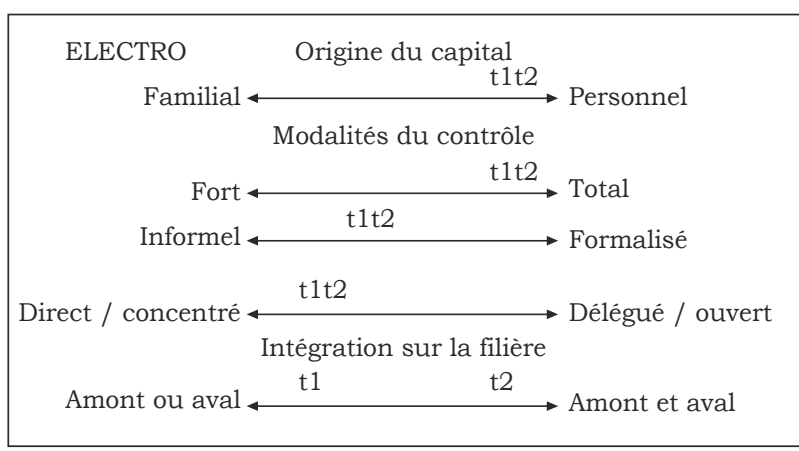

Intégré en amont en 1997 par la création d'un bureau d'études dédié au groupe mais travaillant également en sous-traitance, l'hypogroupe s'est diversifié en aval par le rachat d'un distributeur d'armoires hertziennes qui récupère ainsi la distribution des différents capteurs conçus et produits par l'hypogroupe.

\section{Des dynamiques variées de développement influencées par les trajectoires personnelles mais des constantes dans les logiques d'action}

\section{Un pouvoir de décision qui dépend du type d'hypogroupe}

Il ressort de l'étude que les hypogroupes personnels sont soumis à un pouvoir de décision fort, tandis qu'il est total dans les hypogroupes familiaux : les fonctions-clés de la prise de décision sont «cadenassées » en 1997 comme en 2009. Les hypogroupes personnels ont placé des personnes externes à la tête d'une ou plusieurs filiales. Dans une logique plus managériale, les hypogroupes personnels ont des modes de recrutement plus proches de ceux de la grande entreprise. Le critère de proximité (TORRÈs, 2003), personnelle ou familiale, semble moins prégnant. On observe une formalisation progressive des processus de recrutement du personnel. «Le bouche à oreille si caractéristique des pratiques des TPE s'estompe au fur et à mesure de la croissance de l'entreprise «(Kotey, Slade, 2005), alors qu'on retrouve dans les discours des dirigeants familiaux l'importance des réseaux personnels dans les choix de recrutement (AstLey, VAN dE VEN, 1983).

Entre les deux périodes d'étude, la place du dirigeant reste prédominante au sein des différentes filiales tandis qu'il exerce un contrôle quasi-total dans la holding. Si, dans leurs discours, les dirigeants familiaux attachent une importance presque viscérale à l'indépendance financière, les dirigeants de type personnel manifestent avant tout un souci d'autonomie décisionnelle. Dans les faits, l'observation des structures de capital et des pôles de 
pouvoir dans les différentes sociétés de l'hypogroupe montre que la distinction entre indépendance financière et autonomie de décision se révèle moins essentielle. En effet, dans les quatre cas, l'autonomie de décision passe par une indépendance financière minimale correspondant à la majorité des droits de vote de $51 \%$. Dans les dix hypogroupes étudiés, les filiales sont en grande majorité détenues à $95 \%$ par la holding, voire à $100 \%$. Une répartition astucieuse des droits de vote restants auprès d'associés "fantômes» (épouse, parents, enfants), et un verrouillage des postes-clés dans l'organisation (présidence de conseil d'administration, direction générale dans les sociétés anonymes ou gérance majoritaire dans les SARL par les dirigeantspropriétaires) assurent le dirigeant d'un pouvoir de décision total.

\section{Une formalisation variable des procédures de contrôle}

Dans les hypogroupes familiaux, le contrôle informel de l'activité des filiales par le dirigeant est le mode de contrôle le plus fréquent mais est parfois couplé avec un contrôle formalisé, «pour répondre aux questions du comptable» (BTP). Le contrôle est davantage formalisé dans les hypogroupes personnels, bien qu'il puisse être complété par des contrôles informels. On observe bien ainsi la coexistence durable dans une même entreprise «de divers modes de pilotage locaux sans qu'ils soient coordonnés dans un système de contrôle global et cohérent» (MEyssonnier, ZaWADZKi, 2008).

\section{Quels degrés d'intégration pour quels contrôles?}

Les quatre hypogroupes étudiés exercent tous plusieurs activités, développées à partir d'un même cœur de métier et remplissant des missions différentes. Seul PROMOTEUR a créé une filiale dans un secteur sans lien avec le cœur de métier de l'hypogroupe et met ainsi en évidence l'impact de la trajectoire personnelle du dirigeant. Certains dirigeants ont explicitement recherché à travers le processus d'hypogroupement, la création d'une chaîne d'activités permettant à chaque société de l'hypogroupe de travailler avec une ou plusieurs de ses filiales sœurs, au-delà de l'activité exercée sur le marché (METAL et PROMOTEUR). Chez METAL, «la plus grande partie du chiffre d'affaires de cinq filiales se fait avec les entreprises sœurs, les clients extérieurs venant en renfort si besoin est». Si la filiale exerce une activité complémentaire à celles exercées par ses sociétés-sœurs, c'est la maîtrise d'une plus grande partie du processus du circuit approvisionnement-production-commercialisation qui se trouve renforcée. Dans tous les cas de figure, l'hypogroupe accroît ainsi son contrôle sur son domaine d'activité et réduit sa dépendance envers des partenaires extérieurs. Aucun hypogroupe étudié ne se trouve en situation de dépendance essentielle vis-à-vis d'un partenaire, que ce soit par le volume des approvisionnements ou le chiffre d'affaires lié à un client. On a observé une diversification des sources d'approvisionnements, ou bien une intégration par filialisation du partenaire jugé essentiel ou peu substituable.

La typologie sur continuum rend compte de comportements typés. Les hypogroupes familiaux sont plutôt situés sur la partie gauche en ce qui concerne les modalités du contrôle et ont davantage tendance à s'appuyer sur des modes de contrôle directs et informels et sur des ressources issues du réseau personnel ou familial. Les hypogroupes personnels sont plutôt positionnés sur la partie droite. Leurs procédures de contrôle apparaissent plus formalisées et leurs dirigeants plus ouverts à des opérateurs extérieurs qu'il s'agisse des collaborateurs (délégation) ou des investisseurs. Pour autant, cela ne doit pas remettre en question leur autonomie de décision. Ils apparaissent également plus intégrés sur leur filière d'activités. Au fil du temps, on observe, dans les cas étudiés, une modification de la structure organisationnelle avec des conséquences sur le système de gestion.

\section{* *}

$*$

Avec le temps, le rôle du dirigeant-propriétaire subit des changements d'autant plus forts que l'entreprise d'origine était de faible dimension. L'hypogroupe, comme stratégie alternative de développement de l'entreprise, permet à la fois de limiter les risques liés à la croissance en taille, tels qu'ils sont décrits dans la littérature sur la grande entreprise (perte de maîtrise, distanciation [LECOINTRE, 2010], bureaucratisation [MINTZBERG, 1982, p. 289], perte de réactivité et de flexibilité) et d'atténuer ceux inhérents à la petite taille (risques sociaux, financiers et juridiques, dépendance financière, soumission de la petite entreprise à ses environnements). Pour autant, la modification du domaine de compétences, le passage d'un leadership entrepreneurial à un leadership managérial ne se fait pas toujours sans heurts ni prise de conscience par l'entrepreneur. Certains hypogroupes ont ainsi atteint leur taille maximale en raison des difficultés de leur dirigeant à intégrer ses propres limites, que ce soit en termes d'encadrement ou de capacité à déléguer. Pour d'autres, il semble y avoir eu une prise de conscience de leur «degré de compétence». Les modifications principales semblent surtout avoir été ressenties lorsque le dirigeant a fait évoluer son entreprise du statut de simple entreprise à celui de groupe.

Le domaine de contrôle se trouve lui aussi considérablement élargi. La capacité de délégation du dirigeant, son aptitude à s'entourer de 
collaborateurs, afin de se recentrer sur les aspects les plus stratégiques de la gestion de ses entreprises, ont permis de professionnaliser le mode de management (Flamhotz, 1986). Même si cela semble davantage être le cas dans les hypogroupes personnels, la croissance apparaît «inséparable de la formalisation et de la différenciation » (CYR et al., 2009). De même, Thierry Nobre (2001), dans une étude portant sur les outils de pilotage du contrôle de gestion d'entreprises d'un effectif supérieur à cinquante salariés, constate que la taille est un facteur de contingence explicatif des pratiques de pilotage. L'accroissement de la taille (au-dessus de cent salariés) s'accompagne d'une diversification et d'une complexification des outils de gestion.

Selon les capacités de chaque entrepreneur à savoir s'entourer de compétences lors du développement de son organisation (déléguer et faire confiance, formaliser les procédures de contrôle), la trajectoire organisationnelle sera différente. Le dirigeant peut ainsi choisir de maîtriser sa croissance en taille : dans ce cas, même si le périmètre varie sur la période examinée et si des rachats succèdent aux cessions, au terme de l'étude, le nombre de filiales et leur taille restent proches de ce qu'ils étaient au début. Ou bien, le périmètre de 1'hypogroupe et la taille des filiales s'accroissent et on observe une évolution vers des modes de gouvernance et de contrôle plus proches de ceux de la grande entreprise. La diversité des situations mise en évidence par la typologie sur continuum en fait un outil de lecture pérenne puisqu'il a permis de mettre en lumière aussi bien les pratiques managériales et les modalités du contrôle, fondées sur l'origine du capital (familial ou personnel) que le degré d'intégration dans la filière. La quasi-stabilité de ces structures observée sur dix années peut sans doute expliquer le succès croissant de cette forme organisationnelle. Ce qui pourrait inciter les pouvoirs publics à travailler sur les spécificités de ce type d'organisation et à proposer des outils et aides adaptés. Les raisons de la disparition de deux hypogroupes entre les deux études confortent l'hypothèse de LeVratto (2007, p. 7) selon laquelle, «les microgroupes ne franchissent pas un à un les différents seuils de taille mais sont absorbés par les grands groupes qui leur garantissent l'accès à des ressources...». Pour Marchesnay, les moyennes entreprises tendent à être intégrées, disparaissent ou retrouvent de la compétitivité en devenant plus petites et en se spécialisant, «parva, voire parvula est sed apta, voire aptissima» (petite affaire, voire toute petite, mais apte, voire la plus apte) ${ }^{(12)}$. Mais leur compétitivité repose alors sur les normes « classiques» du management. Comme l'hypofirme, l'hypogroupe réside dans un processus de (re) groupement pour lequel l'objectif essentiel reste de conserver, et surtout de valoriser, les avantages de la petite taille. D'un management entrepreneurial à un leadership managérial, la forme hypogroupe permet donc de pérenniser la petite entreprise.

Le développement de la PME par croissance externe relève d'une logique managériale (grossir), constatée dans les groupes de grande taille (par exemple, des PME agroalimentaires intégrées [contrôlées] ou quasi intégrées [dépendance] par des groupes, ou encore croissance par des regroupements de PME), alors que la croissance par filialisation d'activités existantes relève d'une stratégie entrepreneuriale (éviter de grossir) que l'on observe dans les hypogroupes. Cette dernière apparaît de plus typique du secteur des nouveaux services. $\mathrm{Si}$ l'hypogroupe peut être considéré comme un outil théorique, il n'en reste pas moins que chaque cas empirique témoigne d'une grande singularité, de telle sorte que les bases de leur compétitivité sont aux antipodes de la doxa managériale. Par exemple, la boulangerie artisanale repose sur des facteurs clés de succès tels que la traçabilité des matières, le savoir-faire de l'artisan, les conseils et explications qu'il peut donner à ses clients sur la fabrication et bienfaits de ses produits, à l'opposé de la boulangerie industrielle. Nous rejoignons ainsi les propos de Levratto (2007, p. 2), qui souligne que l'hypogroupe n'est pas seulement une innovation organisationnelle majeure dans le champ des PME mais qu'il «résulte également d'un cadre institutionnel favorable à cette forme de développement». Nombre d'hypogroupes ont pour activités principales des activités de services aux statuts très divers de sorte que tous les travailleurs ne relèvent pas nécessairement du salariat mais d'activités libérales, comme dans les secteurs de la santé et de l'économie sociale et solidaire par exemple. Cet éclatement du statut du salarié offre des perspectives de recherches prometteuses sur la nature spécifique des emplois dans les hypogroupes. 


\section{Bibliographie}

Aubert J.-P., Beaujolin-Bellet R. (2004), «Les acteurs de l'entreprise face aux restructurations : une délicate mutation », Travail et emploi, no 100, pp. 99-112.

Astley G.W., Van De Ven A.H. (1983), «Central perspectives and debates in organization theory", Administrative science quarterly, vol. $28, \mathrm{n}^{\circ} 2$, pp. 245-273.

BARThElEmy J. et al., (1991), Le droit des groupes de sociétés, Paris, Dalloz, coll. «Réussir en Affaires».

BATSCH L. (1993), La croissance des groupes industriels, Paris, Economica.

Basly S. (2006), «Propriété, décision et stratégie de l'entreprise familiale : une analyse théorique», Congrès international de l'AFFI, IAE Poitiers, 26 et 27 juin.

BiRley S. (1986), "Succession in the family firm», Journal of small business management, vol. 24, $\mathrm{n}^{\circ} 3$, pp. 36-43.

Birley S., Westhead P. (1994), «A comparison of new businesses established by "novices" and "habitual" founders in Great Britain», International small business journal, vol. 12, no 1, pp. 38-60.

Bourgeois I. (2007), «Entreprises familiales : un rôle clef outre-Rhin», Regards sur l'économie allemande, no 82 , pp. 31-34.

Bughin C., Colot O. (2008), «La performance des PME familiales belges, une étude empirique» Revue française de gestion, no 186, pp. 1-17.

Chandler A. et al. (1993), «Tightening the lifecycle construct : a taxonomic study of growth stage configurations in high-technology organizations », Entrepreneurship, theory and practice, vol. 18, no 2, pp. 5-30.

Charreaux G., Pitol-Belin J.-P. (1989), «Images et réalités du conseil d'administration », Revue française de gestion, no 74, pp. 51-61.

Churchill N.C., Lewis V.L. (1983), «Les cinq stades de l'évolution d'une PME», Harvard l'Expansion, automne, pp. 51-63.

Cyr A., Meier O., Pacitto J-C. (2009), «La croissance interne des TPE : une tentative d'état des lieux», Actes $\mathrm{du}$ colloque international sur la vulnérabilité des TPE et PME dans un environnement mondialisé, UQTR, Canada.

Colot O., Croquet M. (2005), «La combinaison d'indicateurs comptables et de caractéristiques de propriété permet-elle d'expliquer l'endettement financier des PME familiales?», in Association francophone de comptabilité. Congrès $(26 ; 2005$; Lille), Comptabilité et Connaissance [Ressource électronique].

Colot O., Cropuet M. (2007), «La contribution de variables propres aux $\mathrm{PME}$ et à leur dirigeant dans l'explication de la structure financière des PME», $L a$ revue des sciences de gestion, $\mathrm{n}^{\circ} 228$, pp. 61-72.

Davidsson P., Achtenhagen L., NALdi L. (2004), «Research on small firm growth : a review», Proceedings of the European institute of small growth business.

Debray C. (1997a), Structures et stratégies de l'hypogroupe, thèse NR doctorat ès sciences de gestion, université Montpellier I.

Debray C. (1997b), «L'hypogroupe vecteur facilitateur dans la transmission de la petite entreprise familiale», in Lopez E., Muchnik J. (dir.), Petites entreprises et grands enjeux : le développement agroalimentaire local, Paris, Montréal, l'Harmattan.

Debray C., Leyronas C. (1998), «Réseau et hypogroupe : l'émergence de pratiques managériales en $\mathrm{PME} »$, in Torrès O. (dir.), De nouvelles théories pour comprendre les PME, Paris, Economica, pp. 83-93.

Debray C. (2000), «Réflexions sur les conséquences stratégiques en PME des choix juridiques et fiscaux de la forme de groupe : cas de l'hypogroupe», colloque Journées Nationales des IAE, Biarritz, septembre.

Debray C. (2010), «Les modes de contrôle dans l'hypogroupe : proposition d'une typologie», $X^{e}$ colloque international francophone de la PME, Bordeaux, octobre.

Donckels R., Dupont B., Michel P. (1987), «Multiple business starters : who? Why? What?», Journal of the small business and entrepreneurship, vol. $5 \mathrm{n}^{\circ} 1$, pp. 48-63.

FLAMHOTz E.G. (1986), How to make the transition from entrepreneurship to a professionnally managed firm, San Francisco, Jossey Bass.

Gasse Y., Carrier C. (1992), Gérer la croissance de sa $P M E$, Québec, Les Éditions de l'entrepreneur.

GibB A.A., Sсотт M.G. (1983), «Understanding small firm growth », 6th national small firm policy and research conference, Durham.

Guyon Y. (1994), Droit des affaires, Paris, Economica, coll. «Droit des affaires et de l'entreprise. Série Enseignement», tome 1, 8 e édition.

GODENER A. (2002), «PME en croissance : peut-on prévoir les seuils organisationnels?», Revue internationale $P M E$, vol. 15, no 1, pp. 39-63.

JAUSCH L., Osborn R., MARTIN T. (1980), «Structured content analysis of cases : a complementary method for organizational Research», Academy of management review, vol. 5, no 4, pp. 517-525.

JOHANNISSON B. (1990), «Economies of overview-guiding the external growth of small firms », International small business journal, vol. 9, n 1, pp. 32-44.

Julien P.-A. (dir.), Grepme (Groupe de recherche en économie et gestion des PME) (1994), Les PME : bilan 
et perspectives : ouvrage en l'honneur de Joseph Chica, Cap-Rouge (Québec), les Presses interuniversitaires; Paris, Economica.

Kadjar A. (2007), «Les groupes dans le commerce : une présence croissante», Insee Première, no 1115.

Kolvereig L., Bullvag E. (1992), «Novices versus habitual entrepreneurs : an exploratory investigation», in Birley S., MacMillan I.C. (éd.), Entrepreneurship research, global perspectives. Proceedings of the second annual global conference on entrepreneurship research, London, UK, 9-11 March, 1992, Amsterdam, Elsevier.

Kotey B., Slade P. (2005), «Formal human resource management practices in small growing firms », Journal of small business management, vol. 43, no 1, pp. 16-40.

LeCoIntre G. (2011), Le grand livre de l'économie PME, Paris, Gualino-Lextenso.

Letowski A., Trouve P. (2004), «L'approche typologique : pertinence et identification de sous-groupes au sein des dirigeants de TPE ou la puissance explicative des "modèles d'entreprise"», in Mulliez A., Granger B., Les aides publiques aux TPE, Paris, Commissariat général du plan, pp. 83-124.

Levratto N. (2007), «La PME indépendante et performante, mythe ou réalité? Une analyse fondée sur le phénomène des hypogroupes en France», Revue Internationale PME, vol. 20, no 2, pp. 59-88.

Loiseau H. (2001), «Des groupes de la taille d'une PME», Insee Première, no 764.

Mallard A. (2004), «Les groupes de PME, une structure particulière pour la communication en entreprise», Communication et organisation, consulté le 12 octobre 2012 : http://communicationorganisation.revues. org/2955

Marchesnay M. (1980), «Sur la petite entreprise», Revue d'économie industrielle, vol. 11, no 11, pp. 141-146.

Marchesnay M. (1982), «Pour un modèle d'hypofirme», in Legris G. (éd.), Entreprise et organisations : mélanges en l'honneur de Madame le professeur Jane AubertKrier, Paris, Economica, pp. 70-90.

MARCHESNAY M. (1991), «Del'hypofirme à l'hypogroupe : naissance, connaissance, reconnaissance», Cahiers $d u$ LERASS, no 23, mai, pp. 33-49.

Marchesnay M. (1997), «Petite entreprise et entrepreneur », in Simon Y., Joffre P. (dir.), Encyclopédie de gestion, Paris, Economica, pp. 2209-2219.

MARChESNAY M. (1997), «La moyenne entreprise existet-elle?», Revue française de gestion, no 116, pp. 85-94.

Marchesnay M. (2004), «L'hypofirme, fondement de l'hypermodernité?», 7e CIFEPME, Montpellier, octobre.

Marchesnay M. (2008), «L'hypofirme, vivier et creuset de l'innovation hypermoderne», Innovations, $\mathrm{n}^{\circ} 27$, pp. 147-161.
Mc Millan I.C., Lowe M.B. (1986), «Techniques of the habitual entrepreneur : team building», Snider entrepreneurial center, université de Pennsylvanie, papier de recherche $\mathrm{n}^{\circ} 1$.

Mathe J.-C., Rivet A. (1993), «Décisions stratégiques et structure de propriété des PME», Revue française de gestion, no 95 , pp. 77-85.

Meyssonnier F., ZawadskI C. (2008), «L'introduction du contrôle de gestion en PME : étude d'un cas de structuration tardive de la gestion d'une entreprise familiale en forte croissance», Revue internationale PME, vol. 21, no 1, pp. 69-92.

Mintzberg H. (1982), Structure et dynamique des organisations, Paris, Éditions d'Organisation.

Montmorillon DE B. (1986), Les groupes industriels, analyse structurelle et stratégique, Paris, Economica.

Nobre T. (2001), «Méthodes et outils du contrôle de gestion dans les PME», Finance, contrôle, stratégie, vol. 4, no 2, pp. 119-148.

Observatoire de PME (2005), Dirigeant de PME, un métier? Éléments de réponse, Maisons-Alfort, OSEO services, Paris, diff. La Documentation française, coll. «Regards sur les PME», n 8, 130 p.

Perry C. (1987), «Growth strategies for small firms», International small business journal, vol. 5, no 2, pp. 17-25.

RÉDACTION DES Éditions Francis LeFEBVRE (2006), Groupes de sociétés, 2007-2008: juridique, fiscal, social, Levallois, F. Lefebvre.

Skalitz A. (2002), "Au-delà des entreprises : les groupes », Insee Première, no 836.

ScotT M., Rosa P. (1996), «Opinion : has firm analysis reached its limits? Time for a rethink», International small business journal, vol. 14, no 4, pp. 81-88.

Scott M., Rosa P. (1999), «The prevalence of multiple owners and directors in the SME sector : implications for our understanding of start-up and growth », Entrepreneurship \& regional development, vol. 11, $\mathrm{n}^{\mathrm{o}}$ 1, pp. 21-37.

Starr J.A., Bygrave W.D. (1992), «The second time around : the outcomes, assets and liabilities of prior start-up experience», The Second Global Conference on Entrepreneurship Research, Imperial College, Londres.

Storey, D.J. (1994), Understanding the small business sector, London, Routledge.

Torres O. (2003), «Petitesse des entreprises et gestion du personnel : le rôle central de la proximité», Regards sur les PME, no 3, pp. 58-60.

VAN Loye G. (1991), «Organisation du pouvoir et financement des PME», Revue direction et gestion, no 132-133, pp. 47-62. 
WacheuX F. (1996), Méthodes qualitatives et recherche en gestion, Paris, Economica, coll. «Gestion. Politique générale, finance et marketing».

WAlter G.A., Barney J. B (1991), «Research notes and communications : management objectives in mergers and acquisitions », Small management journal, vol. 11, $\mathrm{n}^{\mathrm{o}} 1$, pp. 79-86.

Wiklund J., Delmar F., Davidsson P. (2003), «What do they think and feel about growth? An expectancy-value approach to small business managers'attitudes towards growth », Entrepreneurship, theory and practice, vol. 27, no 3, pp. 247-269.

Wright M., Robbie K., Ennew C. (1995), «Serial entrepreneurs ", in Bygrave W. et al. (éds.), Frontiers of entrepreneurship research, Babson College, Wellesley.

YIN R.K. (1994), Case study research. Design and methods. Second Edition, Thousand Oaks, Sage Publications. 


\section{Annexe 1:}

Grille d'analyse systémique du processus d'hypogroupement

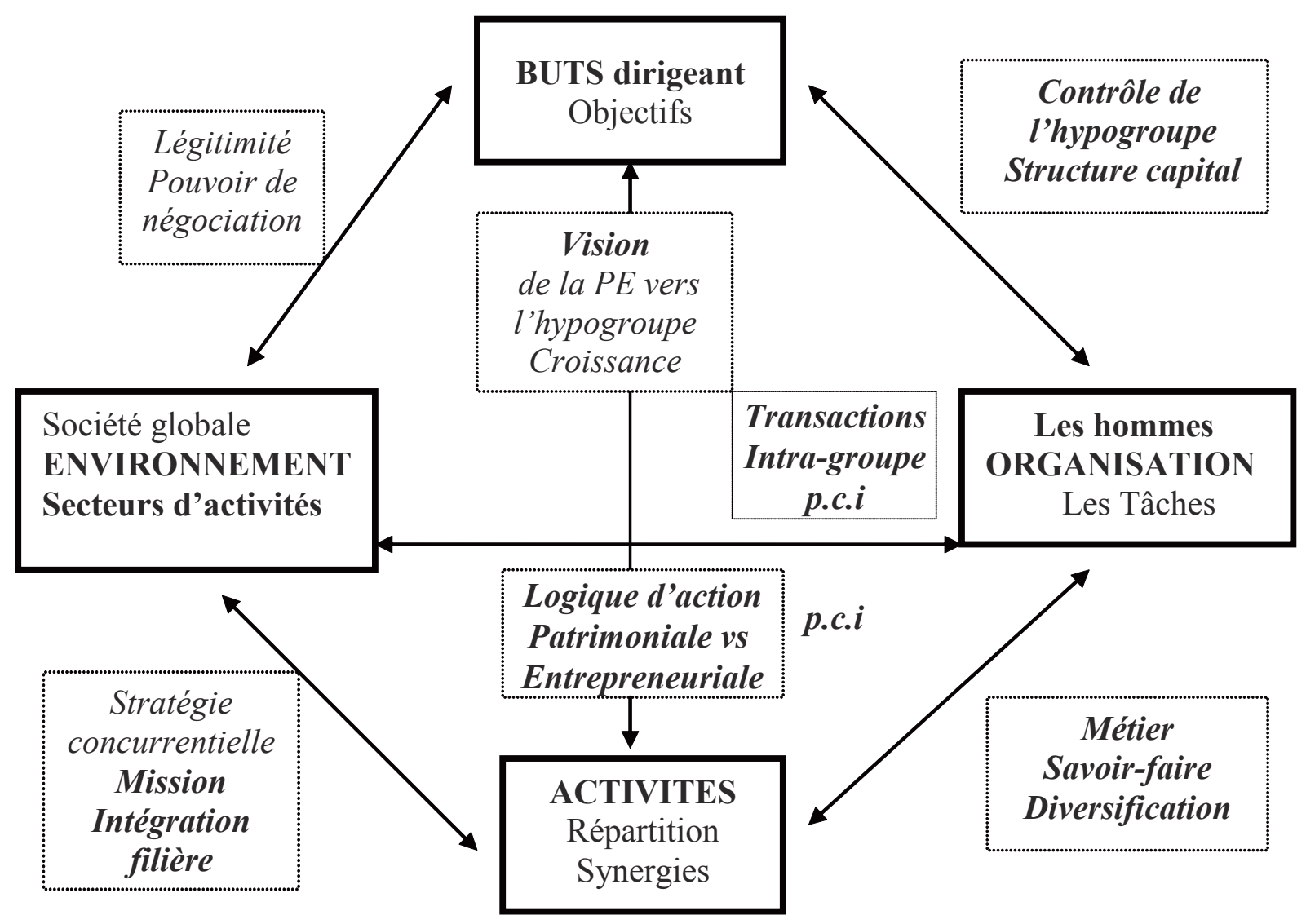

Source : D'après Debray, 1997a. 


\section{Annexe 2 :}

Tableau indicateur de la variable «Contrôle de l'organisation »

\begin{tabular}{|c|c|}
\hline Indicateurs & Indices \\
\hline \multicolumn{2}{|r|}{ Structure du capital } \\
\hline Holding & $\begin{array}{l}\text { Statut, nombre et types d'actionnaires, répartition et type de capitaux } \\
\text { (personnels ou familiaux), place du dirigeant-propriétaire (DP) }\end{array}$ \\
\hline Filiales & Statut, nombre et types d'actionnaires, place du dirigeant-propriétaire (DP) \\
\hline Degré de concentration du capital & $\begin{array}{l}\% \text { détenu par le dirigeant-propriétaire (DP) et les actionnaires } \\
\text { dans la holding et les filiales }\end{array}$ \\
\hline Mécanismes de contrôle & $\begin{array}{c}\text { Statuts juridiques des sociétés, gérance, direction générale } \\
\text { ou présidence du conseil d'administration }\end{array}$ \\
\hline \multicolumn{2}{|c|}{ Pouvoir de décision et contrôle interne du groupe } \\
\hline Direction des filiales & $\begin{array}{l}\text { Dirigeants familiaux ou externes, \% de détention du capital } \\
\text { de la holding ou dans une filiale }\end{array}$ \\
\hline \multirow{2}{*}{$\begin{array}{l}\text { Rôle du CA et nature du contrôle } \\
\text { du dirigeant-propriétaire (DP) }\end{array}$} & $\begin{array}{l}\text { Contrôle total : pouvoir du conseil d'administration nul : pas } \\
\text { d'implication dans le processus stratégique ; pas de révocation ou } \\
\text { d'abaissement de rémunération possible ; asymétrie d'information }\end{array}$ \\
\hline & Contrôle fort : le CA a un rôle faible \\
\hline Holding & $\begin{array}{c}\text { Rôle et pouvoir d'action/sanction du conseil d'administration } \\
\text { sur le dirigeant-propriétaire (DP) }\end{array}$ \\
\hline Contrôle formalisé des filiales & $\begin{array}{l}\text { Présence de documents formalisés de planification (analyse des coûts } \\
\text { et tableaux de bords systématiques) }\end{array}$ \\
\hline Contrôle informel des filiales & $\begin{array}{l}\text { Rareté de documents formalisés/projets de développement, fréquence } \\
\text { élevée d'analyse des coûts, contacts personnels entre le dirigeant- } \\
\text { propriétaire (DP) et les dirigeants des filiales, « réunions de chantier » }\end{array}$ \\
\hline $\begin{array}{l}\text { Style de gestion du } \\
\text { dirigeant-propriétaire (DP) }\end{array}$ & $\begin{array}{c}\text { Concentré, ouvert ; propension à déléguer, attitude envers } \\
\text { les associés minoritaires }\end{array}$ \\
\hline Évolution du style de gestion & $\begin{array}{c}\text { Délégation de certaines tâches, répartition du temps entre gestion } \\
\text { quotidienne et réflexion stratégique, mode de recrutement des } \\
\text { responsables de filiales }\end{array}$ \\
\hline Modes de direction & $\begin{array}{l}\text { Direction directe par supervision directe du dirigeant-propriétaire (DP) } \\
\text { ou direction déléguée (effectuée par un collaborateur familial ou pas) }\end{array}$ \\
\hline Contrôle externe (filière) & \multirow{2}{*}{$\begin{array}{l}\text { Nombre de filiales exerçant dans la filière ; politique de sécurisation } \\
\text { de l'amont et/ou de l'aval }\end{array}$} \\
\hline Intégration & \\
\hline
\end{tabular}

Source : D'après DeBray, 2010. 